\title{
Avaliar materiais de comunicação em saúde melhora literacia em saúde: \\ Tradução e adaptação transcultural do instrumento PEMAT para Portugal
}

Evaluating health communication materials improves health literacy: Translation and cross-cultural adaptation of the PEMAT instrument for Portugal

\section{MARISA BERMUDEZ BRITO}

Unidade de Saúde Familiar Fonte Luminosa, Lisboa, Portugal marisa.brito@arsIvt.min-saude.pt 


\section{RESUMO:}

A comunicação é um aspeto essencial no campo da saúde e oferece um enorme potencial na promoção da literacia em saúde. A literatura científica evidencia que os materiais de educação em saúde, são muitas vezes complexos, não se adequando ao nível de literacia da população a que se destinam. Os profissionais de saúde devem ter conhecimentos e competências neste domínio, e utilizar as melhores práticas disponíveis, já que na maioria das vezes, são responsáveis pela elaboração e disponibilização dos materiais. A existência de instrumentos que permitam avaliar a adequação dos materiais de educação em saúde, ao nível de literacia das pessoas, é uma mais valia para a prática profissional. O instrumento Patient Education Materials Assessment Tool (PEMAT) destina-se à avaliação de materiais de educação em saúde, e inova pela capacidade de avaliar as vertentes da compreensibilidade e acionabilidade. Tendo como objetivo geral, a facilitação de um instrumento que permita avaliar materiais de educação para a saúde, este estudo teve como objetivo específico a tradução e adaptação transcultural do instrumento PEMAT para Portugal. Foi utilizada a metodologia tradução e retroversão e realizado Preteste em amostra por conveniência. A dimensão e a pouca diversificação da amostra constituíram as limitações deste trabalho. A versão final obtida foi considerada muito útil para a prática profissional apresentando-se como um instrumento inovador em Portugal. Espera-se que a sua disseminação permita capacitar os profissionais de saúde na promoção da literacia em saúde dos utentes, à semelhança do que se passa noutros contextos onde o instrumento já é utilizado.

PALAVRAS-CHAVE: PEMAT; comunicação em saúde; literacia em saúde; educação em saúde 


\section{Introdução}

O objetivo geral proposto desta investigação é a disponibilização de um instrumento permitisse avaliar materiais de educação em saúde (MES) enquanto que o objetivo específico foi a tradução e adaptação transcultural do instrumento Patient Education Materials Assessment Tool (PEMAT) para Portugal.

A literatura é consensual com a ideia de que existe um círculo virtuoso onde a comunicação desempenha um papel chave para um melhor empoderamento das pessoas e o empoderamento conduz a uma melhor LS (Almeida, 2019; Belim e Almeida, 2018)

No campo da saúde, a comunicação escrita destaca-se já que impera a cultura da escrita, onde os materiais impressos são largamente utilizados em todos os aspetos dos cuidados de saúde. Espera-se dos utentes que leiam e recordem, aprendam e cumpram as indicações apresentadas, sendo assumido como sua responsabilidade. (Doak, Doak, \& Root, 1996). Exige-se muito aos utentes, esquecendo-se que os profissionais e os sistemas de saúde também têm responsabilidades. É responsabilidade dos PS o fornecimento de informação em saúde, compreensível, que motive e ajude os utentes a aprender, para além de ser culturalmente sensível (Doak, Doak, \& Root, 1996). A questão que se coloca é também esta: Será que os Profissionais de saúde (PS) conseguem responder a todos estes desafios?
Ajudar as pessoas a compreender informação em saúde complexa reduz o risco de resultados negativos. Apesar de muitas vezes reforçarem a educação em saúde verbal com recurso a informação escrita, os enfermeiros á semelhança de outros PS sobrestimam a literacia em saúde (LS) das pessoas (Dickens, Lambert, Cromwell, \& Piano, 2013). Sabendo que todas as pessoas beneficiam de informação em saúde simples e fácil de ler, para a melhoria contínua da compreensão e dos resultados em saúde, os enfermeiros devem compreender e aplicar os princípios da literacia no desenvolvimento de MES (Foster, Idossa, Mau, \& Murphy, 2016).

Apesar de existirem diretrizes que auxiliam a elaboração e desenvolvimento de MES, a pesquisa bibliográfica efetuada revelou a ausência, em Portugal, de instrumentos que auxiliem os PS na avaliação dos mesmos, pois não há a cultura de validação e simplificação de folhetos em Portugal. Emerge assim a necessidade do desenvolvimento de ferramentas realizadas no contexto português que possam avaliar a inteligibilidade, a qualidade de conteúdos e a adequação à LS dos utentes promovendo assim a compreensão e capacitação dos mesmos (Rosendo \& Santiago, 2017), pelo que este trabalho se apresentou como uma oportunidade de colmatar essa carência. 


\section{Enquadramento teórico}

\section{Literacia em saúde e a dimensão da compreensão}

A Literacia em saúde (LS) implica que o indivíduo seja capaz de descrever e comunicar sintomas físicos e mentais, compreender as orientações dos profissionais, tomar decisões acerca de tratamentos médicos e do quando e como procurar ajuda médica, compreender instruções e prescrições, folhetos médicos, de consentir de forma livre e esclarecida entre outras (Carmo, 2016). São assim, exigidas múltiplas competências às pessoas, especificamente relacionadas com o acesso, compreensão e uso da informação em saúde (Sørensen et al., 2012) num ambiente de saúde, que é caraterizado por ser complexo, com uma linguagem técnica muito própria e cada vez mais tecnológico.

Estima-se que em Portugal, 60\% da população portuguesa apresenta um nível de literacia baixo ou inadequado e que existem grupos mais vulneráveis no campo da LS, nomeadamente: indivíduos com 66 ou mais anos, com baixos níveis de escolaridade, com rendimentos até 500€, com doenças prolongadas, com uma autoperceção de saúde "má"; que frequentaram no último ano 6 ou mais vezes os cuidados de saúde primários, que se sentem limitados por terem alguma doença crónica (Espanha, Avila \& Mendes, 2016). Também McClure, Ng, Vitzhum \& Rudd (2016) incluem no grupo de pessoas com baixa LS as que vivem na pobreza e em áreas com recursos escassos, membros de minorias e as populações imigrantes.

As pessoas com baixa literacia saltam as palavras que não compreendem, não fazem categorias, não conseguem no final juntar ou interpretar o sentido. Mesmo as pessoas com elevada literacia preferem textos mais simples (Vaz de Almeida, 2016).

Sabe-se as pessoas com baixa literacia são desincentivadas pelo aspeto global da informação em saúde, bem como pelo seu conteúdo, quando a informação relevante é difícil de encontrar e de perceber (van Beusekom, Grootens-Wiegers, Bos, Guchelaar, \& van den Broek, 2016).

Segundo Pedro, Amaral \& Escoval (2016) as pessoas com baixa LS apresentam uma menor probabilidade: de perceber a informação escrita e oral dada pelos PS, de conseguirem navegar no sistema de saúde, de acatarem prescrições e de realizar procedimentos necessários.

As pessoas com baixa LS tendem a ser passivas nas consultas médicas e a gerir com menos eficácia a sua saúde (Rademakers \& Heijmans, 2018). Os aspetos referidos devem ser tomados em conta já que segundo Belim e De Almeida (2018) para as pessoas com baixa LS, as exigências de comunicação devem redobradas e a comunicação com elementos específicos pode aumentar a sua LS. 


\section{Comunicação em saúde promotora da literacia em saúde}

A comunicação em saúde diz respeito ao estudo e utilização de estratégias de comunicação para informar e para influenciar as decisões dos indivíduos e das comunidades no sentido de promoverem a sua saúde (Carvalho Teixeira, 1999). Assim a comunicação em saúde pode promover a LS já que está relacionada com uma maior consciencialização dos riscos e motivação para a mudança de comportamentos; facilita as escolhas complexas em saúde e nas doenças; melhora a adaptação a doença e qualidade de vida; melhora comportamentos de adesão e de procura de cuidados (Carvalho Teixeira, 1999). As competências em comunicação são vitais para a otimização da relação terapêutica, e para a LS, já que melhores resultados em saúde e baseiam-se na capacidade de comunicar com as pessoas (Belim \& De Almeida, 2018).

A informação disponível muitas vezes não satisfaz as necessidades dos indivíduos, seja por ser haver excesso de informação, por ser demasiado generalista, estar pouco orientada para a resolução de problemas práticos, usar uma linguagem técnica e complexa ou até em alguns casos estar desatualizada. $\mathrm{O}$ material informativo deveria ser facilitado em suportes distintos e adaptar-se as necessidades das populações mais vulneráveis. Com frequência a informação é transmitida por escrito, muitas vezes através de folhetos impressos e o problema pode surgir quando estes materiais são difíceis de entender para a população.
Desta forma é impossível que cumpram o objetivo para o qual foram desenhados, que é melhorar os conhecimentos das pessoas, a sua participação e por último os seus comportamentos saudáveis (Barrio, SimónLorda, Melguizo, \& Molina, 2011).

Apesar da atenção crescente sobre estas questões, sabe-se que a maioria dos MES são inadequados ou redigidos acima dos níveis de leitura recomendados pelos peritos (Stossel, Segar, Gliatto, Fallar, \& Karani, 2012). Muitos documentos foram escritos com níveis de legibilidade acima da média da população e muitos utilizam um excessivo número de palavras difíceis ou termos técnicos que comprometem sua compreensão (Mialhe \& Cristiane, 2008). Assim muitos utentes serão incapazes de compreender a informação fornecida e agir de acordo com o plano de tratamento prescrito. Podem também ter dificuldades em preparar-se para exames, procedimentos ou cirurgias (Parnell, 2015). O que vai de encontro ao referido por van Beusekom, Grootens-Wiegers, Bos, Guchelaar, \& van den Broek (2016) quando assinalam que apesar dos folhetos impressos serem ainda os meios preferidos para receber instruções sobre medicação, os materiais existentes são inadequados, o que faz com que as pessoas com baixa literacia dependam de outras fontes de informação cuja fiabilidade é variável como por exemplo amigos e familiares ou informação online (van Beusekom, Grootens-Wiegers, Bos, Guchelaar, \& van den Broek, 2016). 
Mialhe e Cristiane (2008) referem que os "problemas como o estilo de linguagem utilizado, a legibilidade do material, o conteúdo e forma de apresentação, entre outros, podem interferir no processo de comunicação (p. 81). Os investigadores acrescentam que se os pacientes não conseguirem compreender as informações nestes materiais impressos, "o seu uso será limitado ou inexistente" (Mialhe \& Cristiane, 2008, p. 82)

Os MES disponíveis nas salas de espera dos médicos de família não promovem a LS de uma proporção significativa das pessoas. Informação desenvolvida de forma rigorosa, boa e acessível tem o potencial de informar, educar e empoderar as pessoas a tomarem decisões em saúde e na gestão da mesma. Para tal, os MES, devem possuir níveis de legibilidade adequadas às audiências a que se destinam. Quando a informação está acessível apenas para uma proporção das pessoas com competências de leitura mais avançadas, não faz nada para reduzir as desigualdades na saúde, e talvez até sirva para as aumentar (Protheroe, Estacio, \& Sady-Khan, 2015). Melhorar a compreensão dos processos de doença, da prevenção e tratamento é um passo necessário para a melhoria dos resultados em saúde. Devido ao enorme impacto da LS no sistema de saúde (...) é crucial que o fornecimento de MES concebidos para a compreensão para pessoas de todos os níveis de literacia (Stossel, Segar, Gliatto, Fallar, \& Karani, 2012). Uma das formas de mitigar os efeitos nefastos da baixa LS é assegurar que o
MES são fáceis de aceder, processar, compreender e aplicar, por outras palavras que as exigências em LS do material não excedam as competências em LS das pessoas (Cajita, Rodney, Xu, Hladek, \& Han, 2017) (Keçeci, Toprak, \& Seçil, 2017).

Os MES bem concebidos podem melhorar a capacidade de um utente gerir um plano de tratamento prescrito ou uma doença crónica (Parnell, 2015). A comunicação em saúde tem de ser clara, compreensível, recordável, credível, consistente ao longo do tempo, baseada na evidencia, personalizada, feita "à medida" das necessidades de informação do utente naquele momento, adaptado ao seu nível cultural e estilo cognitivo (Carvalho Teixeira, 1999).

É imperativo que os PS adaptem a sua prática para utilizar estratégias que comprovadamente respeitem as necessidades de comunicação dos utentes, ou seja de forma a que estes compreendam e usem a informação (Johnson, 2015-2016). Neste contexto existe a necessidade de recomendações para que os PS especialmente os enfermeiros já que usam mais frequentemente os MES, recebam formação acerca das fórmulas relevantes e dos pontos a considerar aquando da utilização e no desenvolvimento de MES (...) acentuando que existe falta de formação dos enfermeiros no desenvolvimento de MÊS, assim como uma ausência de normas escritas que definam as guidelines para a preparação destes materiais (Keçeci, Toprak, \& Seçil, 2017). 
Instrumento Patient Education Materials Assessment Tool (PEMAT)

Em Portugal, não há uma cultura de validação e simplificação dos folhetos, havendo poucos estudos publicados de validação, avaliação e simplificação de materiais escritos. Este tipo de procedimento deve ser valorizado para melhorar a efetividade da informação escrita (Rosendo \& Santiago, 2017). Para melhorar a comunicação entre os sistemas de saúde e os utentes recomenda-se desenhar a informação de acordo com as caraterísticas da população. Com este objetivo a Agência Europeia de Avaliação dos Medicamentos elaborou os requisitos de legibilidade que devem cumprir as bulas dos medicamentos. Igualmente o Manual de Educação Sanitária nos cuidados primários de saúde da OMS, recomenda que as mensagens de saúde se transmitam de forma legível. Existe uma grande variedade de catálogos de recomendações para melhorar a legibilidade dos folhetos (Barrio, SimónLorda, Melguizo, \& Molina, 2011).

De acordo com Shoemaker, Wolf \& Brach (2014) são abundantes os estudos que avaliam a legibilidade, a adequação ou a inteligibilidade de MES numa multiplicidade de tópicos, e a evidência é clara que a maioria dos MES são demasiado complexos para utentes com baixa LS.

A legibilidade é apenas um dos fatores que influenciam a compreensão da informação escrita. Outras características de um documento, tais como a escolha de palavras, a organização, a apresentação, podem afetar a compreensão das pessoas (Brega, et al., 2015). Em 2013 a Agency for Healthcare Research and Quality (AHRQ), desenvolveu através de uma equipa de pesquisadores em colaboração com um painel de peritos em LS, em criação de conteúdos, em educação para a saúde e em comunicação, o instrumento PEMAT. Este foi desenvolvido para colmatar as limitações existentes quando apenas se utilizam de fórmulas de legibilidade na avaliação de MES (Vishnevetsky, Walters, \& Tan, 2017).

O PEMAT é um método validado de avaliação de MES. Um documento é considerado compreensível quando as pessoas conseguem processar e explicar as mensagens chave. Os materiais são considerados acionáveis quando as pessoas conseguem identificar as ações que podem realizar de acordo com as informações fornecidas (Shoemaker, Wolf, \& Brach, 2014).

O PEMAT utiliza um total de 26 itens para avaliar a compreensibilidade e acionabilidade de materiais impressos ou audiovisuais. Cada item reflete uma recomendação para desenvolver documentos que sejam fáceis de compreender e de acionar. As pontuações totais para a compreensibilidade e acionabilidade representam a percentagem de recomendações a que um documento adere. Este instrumento constitui um método sistemático de avaliação e comparação de MÊS e foi elaborado para ser utilizado por PS, servindo como guia na elaboração MES, permitindo determinar se os utentes são capazes de compreender a informação 
disponibilizada e agir de acordo com a mesma (Shoemaker, Wolf, \& Brach, 2013).

O PEMAT consiste em 26 itens e duas escalas: compreensibilidade (19 itens) e acionabilidade (7 itens). Existem duas versões: o PEMAT-P (ANEXO 1) para materiais impressos (compreensibilidade $=17$ itens $\mathrm{e}$ acionabilidade $=7$ itens) e o PEMAT-A/V (ANEXO 2) para materiais audiovisuais (compreensibilidade $=13$ itens $\mathrm{e}$ acionabilidade $=4$ itens). O PEMAT pontua os materiais numa escala de 0-100 (Shoemaker, Wolf, \& Brach, 2014).

No desenvolvimento do PEMAT foi estabelecido um limite de $70 \%$ para o que deveria ser considerado um material compreensível ou acionável, ou seja, uma pontuação no PEMAT de $70 \%$ ou inferior seria considerada pouco compreensível ou pouco acionável (Shoemaker, Wolf, \& Brach, 2014).

Enquanto há poucos anos a única forma das pessoas receberem informação em saúde era através da interação direta com os PS, as tecnologias de informação alteraram esta situação de forma dramática. De facto, nas últimas décadas, e sobretudo durante 2020, desde o início da pandemia COVID -19, assistimos a uma migração constante do consumo de informação dos meios materiais e físicos, para os espaços e meios online, onde a informação se torna mais dinâmica (Arsenault, Blouin, \& Guitton, 2016). O PEMAT é o único instrumento que inclui uma avaliação objetiva de materiais audiovisuais (Vishnevetsky, Walters, \& Tan, 2017).

O PEMAT é um instrumento que não requer treino, podendo ser usado por diferentes profissionais como clínicos, educadores em saúde, bibliotecários em saúde e até leigos, na identificação de MES que contribuem para melhorar a literacia de saúde das pessoas (Shoemaker, Wolf, \& Brach, 2014).

De acordo com o Guia de utilização deste instrumento, os MES são considerados 1) compreensíveis: quando os utentes de diferentes contextos e níveis de literacia em saúde, conseguem processar e explicar os pontos-chave, e; 2) acionáveis: quando os utentes de diversos contextos e níveis de literacia em saúde conseguem identificar o que têm de fazer, baseados na informação apresentada (Shoemaker, Wolf, \& Brach, 2013). (Figura 1) 


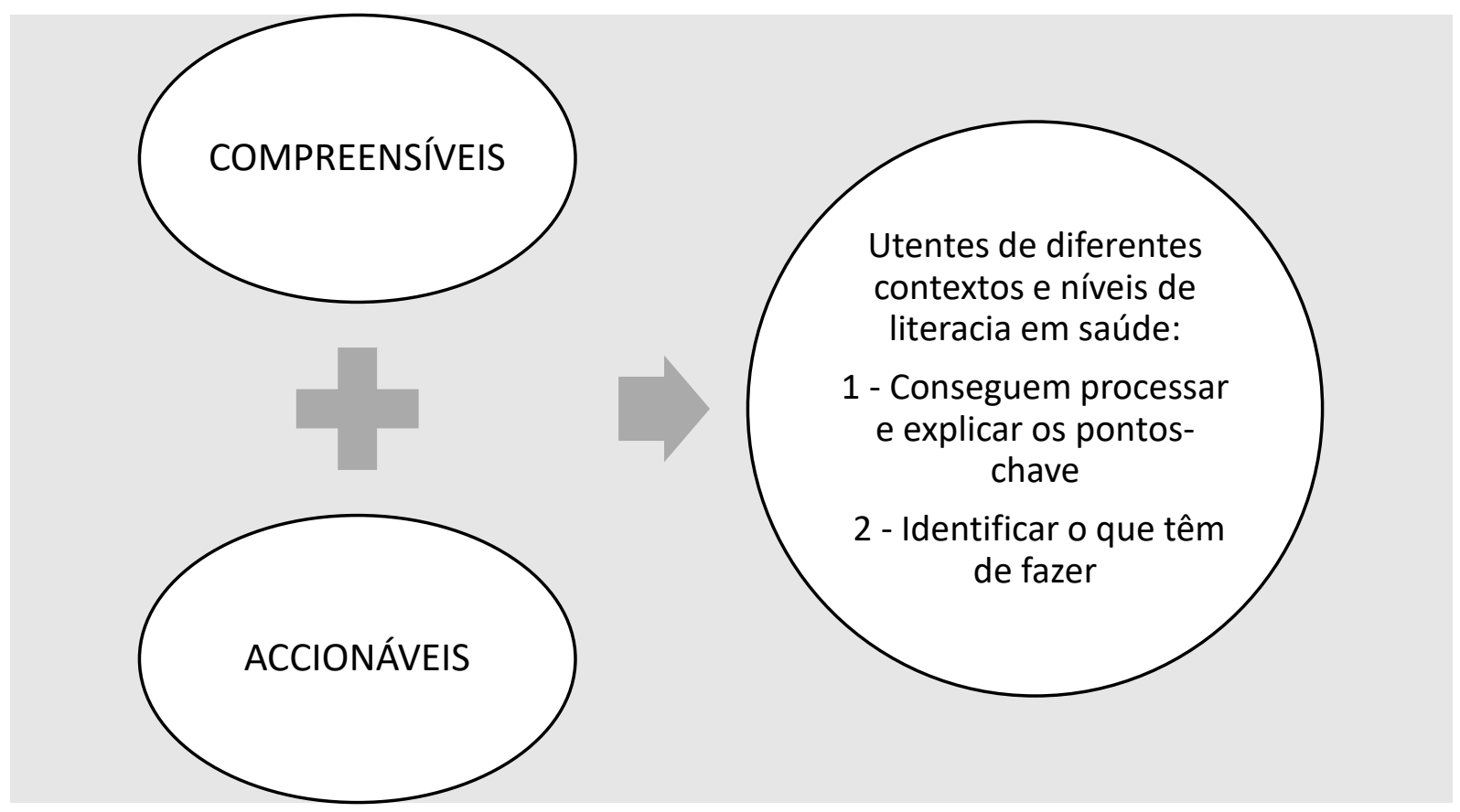

Fonte: Elaboração própria

O PEMAT foi sujeito a vários testes de fiabilidade e aperfeiçoamento e demonstrou ser um instrumento fiável, com consistência interna e evidência na validade de construto podendo ser utilizado para avaliar a compreensibilidade e acionabilidade de materiais escritos e audiovisuais em diferentes tópicos. Assim, o PEMAT cumpre o objetivo de desenvolver e disseminar informação em saúde que seja segura, precisa, acessível e acionável de acordo com o Plano Nacional de Ação para Melhoria da Literacia em Saúde americano. (Shoemaker, Wolf, \& Brach, 2014).

No entanto, o PEMAT apresenta algumas limitações pois nem todos os materiais que apresentem pontuações elevadas para o PEMAT, são de alta qualidade. O PEMAT não avalia, por exemplo, a precisão da informação apresentada, isto é, o rigor, a relevância e o detalhe da mesma, assim como não avalia a legibilidade da informação apresentada, ou seja, a facilidade de leitura. Por exemplo, um material pode ser bastante compreensível, mas apresentar informação incorreta. Nestes casos, será vantajoso usar em conjunto com o PEMAT, outras ferramentas que colmatem as suas limitações. O guia de utilização do instrumento recomenda a realização de um teste de legibilidade para materiais impressos, em conjunto com a utilização do PEMAT. A utilização apenas de fórmulas de legibilidade, não substitui a utilização do PEMAT, já que ignoram fatores que contribuem para a compreensão e podem ser enganadoras (Shoemaker, Wolf, \& Brach, 2013). 
O PEMAT não avalia a legibilidade, sendo recomendado que se conjugue uma avaliação da legibilidade em associação ao PEMAT. No entanto, a dificuldade na leitura tem sido consistentemente associada a uma pior compreensão e pode ser facilmente e objetivamente analisada (Shoemaker, Wolf, \& Brach, 2014).

Este instrumento já foi aplicado em vários em estudos internacionais quer isoladamente (Wong, Keefe, Gilad, Chong-Sun Li, \& Levi, 2017) (Han, Grisales, \& Sridhar, 2018) (Schorr, Hunter, \& Zuzelo, 2018) (Zellmer, Zimdars, \& Safdar, 2015) como em conjunto com outros instrumentos (Brutting, et al., 2018) (Harris, et al., 2018) (Keçeci, Toprak, \& Seçil, 2017) (Kirby, Reynolds, Walker, Furer, \& Pryor, 2018) (McClure, Ng, Vitzhum, \& Rudd, 2016).

Estão disponíveis duas versões separadas para a utilização em materiais impressos e materiais audiovisuais. A versão para materiais impressos é indicada para materiais impressos como brochuras, panfletos, etc. apresentando 17 itens para a compreensibilidade e 7 itens para a exequibilidade. A versão para materiais audiovisuais é indicada para vídeos, materiais multimédia, etc. e compõe-se de 13 itens para a compreensibilidade e 4 itens para a exequibilidade. Apesar da maioria dos itens do PEMAT ser relevante para ambos as versões dos materiais, alguns itens são apenas aplicáveis a um tipo de material. Assim, a numeração dos itens para a não é consecutiva pois em cada versão são omitidos os itens não relevantes. Assim, a versão para materiais impressos inclui os itens 1-12 e 15-19 para a compreensibilidade e os itens $20-26$ para a acionabilidade. Já a versão para materiais audiovisuais inclui os itens 1, 3-5, 8-14 e 18-19 e para a acionabilidade 20-22 e 25. (Quadro 1).

Quadro 1. Versões do PEMAT

\begin{tabular}{|c|c|c|}
\hline TIPO DE MATERIAIS & INDICAÇÃO & $\mathbf{N}^{0}$ DE ITENS \\
\hline Materiais impressos & brochuras, panfletos, etc. & $\begin{array}{l}17 \text { itens para a } \\
\text { compreensibilidade } \\
7 \quad \text { itens para a } \\
\text { exequibilidade }\end{array}$ \\
\hline Materiais audiovisuais & $\begin{array}{l}\text { vídeos, materiais } \\
\text { multimédia, etc. }\end{array}$ & $\begin{array}{l}13 \text { itens para a } \\
\text { compreensibilidade } \\
4 \quad \text { itens para a } \\
\text { exequibilidade }\end{array}$ \\
\hline
\end{tabular}


Para além deste instrumento, foi ainda desenvolvido um guia de utilização do mesmo, que descreve o desenvolvimento do PEMAT, os seus objetivos e inclui informações relevantes para a utilização do instrumento. $\mathrm{O}$ guia analisa todos os 19 itens que avaliam a compreensibilidade e os 7 itens que avaliam a acionabilidade, esclarecendo dúvidas que possam surgir na avaliação (Shoemaker, Wolf, \& Brach, 2013).

\section{Metodologia}

O processo de tradução e adaptação do PEMAT para Portugal, iniciou-se com a solicitação de autorização aos autores do instrumento original (AHRQ - Agency of Healthcare Research and Quality) em 2018, para tradução e adaptação para Portugal, a qual foi concedida por David Lewin (AHRQ).

Seguiu-se o processo de tradução/retroversão e adaptação transcultural do instrumento, com o objetivo de obter um instrumento equivalente ao desenvolvido no país de origem, assegurando-se a equivalência semântica e de conteúdo, sendo adotada para tal a metodologia proposta por Beaton, Bombardier, Guillemin \& Ferraz (2000). Os autores referidos sugerem como etapas no processo de adaptação cultural: a tradução, a síntese das traduções, a retroversão, a revisão por grupo de peritos, o Preteste, o envio do material produzido aos autores do instrumento original e por fim a avaliação das propriedades psicométricas do instrumento adaptado (Beaton, Bombardier, Guillemin, \& Ferraz, 2000).
Assim, o procedimento foi desenvolvido de acordo com os seguintes passos: realização de duas traduções independentes do instrumento, para português; confronto e análise das duas traduções por PS, para obtenção da primeira versão em português; retroversão por tradutores independentes especialistas em comunicação, não conhecedores da versão inicial em língua inglesa e avaliação por grupo de peritos e Preteste.

\section{Resultados}

Relativamente à tradução, esta foi realizada por dois tradutores, um dos quais era PS e perito em LS, portanto conhecedor dos conceitos abordados, enquanto o outro tradutor desconhecia os objetivos do estudo e não apresentava formação na área da saúde de acordo com a metodologia proposta por Beaton e colegas (Beaton, Bombardier, Guillemin, \& Ferraz, 2000).

As traduções foram confrontadas para obter uma primeira versão em português. Na etapa seguinte, foi realizada a retroversão da versão obtida, ou seja, o instrumento foi traduzido de português para inglês por dois tradutores independentes, desconhecedores da versão original do instrumento. A síntese das duas versões retrovertidas apresentou uma proximidade bastante satisfatória com a versão original do instrumento.

A avaliação por comité de especialistas foi a etapa seguinte tendo sido sugeridas ligeiras 
melhorias na linguagem para evitar interpretações dúbias.

Na etapa seguinte foi realizada o Preteste, para tal foi selecionada uma amostra por conveniência composta por PS com interesse e formação em LS. Foram convidados 17 participantes, via email, sendo contextualizado o trabalho em desenvolvimento, as instruções para participação, pedido o consentimento e enviadas em anexo, as versões preliminares para materiais impressos e para materiais audiovisuais para avaliação.

Acederam participar 6 PS que eram maioritariamente do género feminino (83\%) com uma média de idade de 33 anos. Os participantes avaliaram individualmente as versões do instrumento desenvolvido com vista á avaliação semântica, verificação da adequação e clareza das instruções, dos itens, das opções de resposta bem como da facilidade ou dificuldade na compreensão dos termos utilizados. Pediu-se que analisassem os itens, tendo em conta eventuais erros, desadequações na formulação dos mesmos e pedindo-se sugestões alternativas.

Houve um consenso entre os participantes relativamente à importância e utilidade prática do instrumento desenvolvido no contexto da saúde em Portugal, pela capacitação dos PS relativamente ao desenvolvimento e seleção de MES de alta qualidade e desse modo promover a LS das pessoas. Foi destacada a fácil compreensão do instrumento, com a utilização de linguagem clara e acessível e evidenciado o fácil preenchimento do instrumento.
Foram realizadas alterações em alguns dos itens. Relativamente à versão para materiais impressos no Item 2. O material não inclui informação ou conteúdo que distraia do seu objetivo e Item 7. O material não exige que o utilizador faça cálculos de acordo com um dos participantes, o fato das frases se encontrarem formuladas na negativa poderia induzir erro na resposta.

Os autores do instrumento, estabeleceram como regra, para as opções de resposta, que a opção discordo seria sempre cotada como 0, e a opção concordo com 1. Visto que a sugestão realizada implicaria uma alteração nessa regra, não foi introduzida na versão final do instrumento.

No Item 4. Os termos médicos são utilizados apenas para familiarizar a audiência com os mesmos. Quando usados, os termos médicos são definidos foi observado por dois dos participantes que os MES utilizam inevitavelmente termos médicos e que estes muitas vezes são centrais à compreensão de determinadas situações clínicas ou tratamentos. Dando como exemplo, as palavras "TAC - tomografia axial computorizada" ou "nervo" que são de difícil tradução em linguagem acessível e clara. Esta observação vai de encontro à recomendação proposta no estudo desenvolvido por Vishnevetsky, Walters \& Tan (2017) que avalia as propriedades do PEMAT, o instrumento original. Assim, o item foi modificado para Item 4. Os termos médicos utilizados são necessários para compreender a situação 
clínica ou o tratamento apresentado no material, sendo definidos inicialmente.

O estudo referido realiza também uma recomendação relativa ao Item 5. O material usa voz ativa, referindo que deveria constar um alerta para assinalar a opção Concordo, se a caraterística ocorrer em $80 \%$ do tempo. Esse item foi também apontado por 4 dos participantes, que referiram ser vantajosa a inclusão da definição de voz ativa e de um exemplo da mesma, já que utilizadores leigos podem apresentar dificuldade na compreensão deste item. Assim, foi incluída a definição e um exemplo de voz ativa, bem como a recomendação apresentada no estudo mencionado.

Por ser bastante pertinente foi também incluída outra recomendação, exposta pelo estudo referido, nomeadamente em relação ao Item 6 . Os números que aparecem no material são claros e fáceis de entender, que refere que se deve escolher a opção de resposta Não Aplicável, não apenas no caso de não existirem números, mas também caso os números se refiram a horas, datas, números de telefone ou moradas, pelo que foi colocada na versão final do instrumento.

Em relação aos Item 15. O material utiliza, sempre que necessário, ajudas visuais, que tornam o conteúdo mais facilmente compreensível (ex.: ilustração do tamanho de uma porção saudável), e ao Item 23. O material fornece ferramentas específicas (ex.: um plano de refeições, uma lista) sempre que possam ajudar o utilizador a realizar ações, e
Item 26. O material utiliza ajudas visuais, sempre que estas tornem mais fácil, realizar uma ação de acordo com as instruções ou informação apresentadas, os dois participantes referiram que deveriam constar como opção de resposta Não Aplicável, caso o material em avaliação não apresentasse ajudas visuais (itens 15 e 26) ou ferramentas (item 23). Estes dados vão de encontro às recomendações referidas no estudo desenvolvido por Vishnevetsky, Walters \& Tan (2017) que referem que a expressão sempre que utilizada na formulação desses itens pode ser causar confusão aos avaliadores por ser demasiado subjetiva, para além das dúvidas apresentadas na avaliação do material, caso este não apresentasse ajudas visuais ou ferramentas, mas estas não fossem consideradas necessárias. Assim foi adicionada a opção de resposta Não aplicável aos itens 15, 23 e 26.

$\mathrm{Na}$ versão para materiais audiovisuais e relativamente ao Item 14. O material permite que o utilizador oiça as palavras de forma clara (ex.: não muito rápido, não distorcido) um dos participantes ressalvou que os exemplos estavam formulados na negativa, e poderiam, por isso, causar dúvidas. Deram a sugestão de incluir como opção "Item 14.O material permite que o utilizador oiça as palavras de forma clara (ex.: com velocidade, volume e clareza sonoras adequadas à sua compreensão), a qual foi aceite e integrada na versão final.

O mesmo participante e relativamente ao Item 18. O material usa ilustrações e fotografias nítidas e simples anotou que as palavras 
ilustrações e fotografias podiam não ser adequadas a todos os materiais audiovisuais, como vídeos, por exemplo, pelo que foram substituídas pela expressão ajudas visuais. Neste item foi mencionado também que certas caraterísticas do material, como a qualidade da imagem ou a focagem podem afetar a compreensão do mesmo, e que deveriam constar no item, pelo que foi modificado ficando Item 18. O material usa ajudas visuais nítidas e simples (ex.: com qualidade $e$ focagem adequadas à sua compreensão).

Após a análise dos comentários dos participantes, o instrumento foi revisto tendo sido realizadas as correções e adaptações necessárias, objetivou-se que estas análises permitissem a obtenção da versão final o instrumento. Foram assim, elaboradas as versões finais para materiais impressos para Portugal. (Quadro 2, 3 e 4).

\section{Quadro 2. - PEMAT - Materiais impressos: Compreensibilidade}

Instrumento para Avaliação de Materiais de Educação em Saúde - Versão para Materiais Impressos

Título do Material

Data:

\section{Compreensibilidade}

\begin{tabular}{|c|c|c|c|}
\hline N.2 2 & Item & Opçত̄es de resposta & Classificação \\
\hline \multicolumn{4}{|c|}{ Tópico: Conteúdo } \\
\hline 1. & É claramente evidente o objetivo do material & Discordo $=0$, Concordo $=1$ & \\
\hline 2. & O material não inclui informação ou conteúdo que distraia do seu objetivo & Discordo $=0$, Concordo $=1$ & \\
\hline \multicolumn{4}{|c|}{ Tópico: Estilo e escolha de palavras } \\
\hline 3. & O material utiliza linguagem comum, do dia a dia & Discordo $=0$, Concordo $=1$ & \\
\hline 4. & $\begin{array}{l}\text { Os termos médicos utilizados são necessários para compreender o tema apresentado no } \\
\text { material, sendo definidos inicialmente }\end{array}$ & Discordo $=0$, Concordo $=1$ & \\
\hline 5. & O material usa voz ativa ${ }^{1}$ & Discordo $=0$, Concordo $=1$ & \\
\hline \multicolumn{4}{|c|}{ Tópico: Uso de números } \\
\hline 6. & Os números que aparecem no material são claros e fáceis de entender & $\begin{array}{l}\text { Discordo }=0 \\
\text { Concordo }=1 \\
\text { Sem números ou números } \\
\text { como datas, horas, } \\
\text { telefones e moradas= N/A }\end{array}$ & \\
\hline 7. & O material não exige que o utilizador faça cálculos & $\begin{array}{l}\text { Discordo }=0 \\
\text { Concordo }=1 \\
\text { Sem números= N/A }\end{array}$ & \\
\hline \multicolumn{4}{|c|}{ Tópico: Organização } \\
\hline 8. & O material reparte a informação em pequenas secções & $\begin{array}{l}\text { Discordo }=0, \text { Concordo }=1 \\
\text { Material muito } \text { curto }^{2}=\mathrm{N} / \mathrm{A}\end{array}$ & \\
\hline 9. & As secçōes do material têm cabeçalhos informativos & $\begin{array}{l}\text { Discordo }=0, \text { Concordo }=1 \\
\text { Material muito } \text { curto }^{2}=\mathrm{N} / \mathrm{A}\end{array}$ & \\
\hline 10. & O material apresenta a informaç̃o em sequência lógica & Discordo $=0$, Concordo $=1$ & \\
\hline 11. & O material contém um resumo & Discordo $=0$, Concordo $=1$ & \\
\hline
\end{tabular}

${ }^{1} \mathrm{Na}$ voz ativa, o sujeito pratica a ação expressa pelo verbo. Assim, a pessoa é abordada diretamente (Ex.: Coma frutas e verduras)

${ }^{2}$ Um material muito curto é definido como material que apresente dois ou menos parágrafos e não mais de uma página de comprimento 


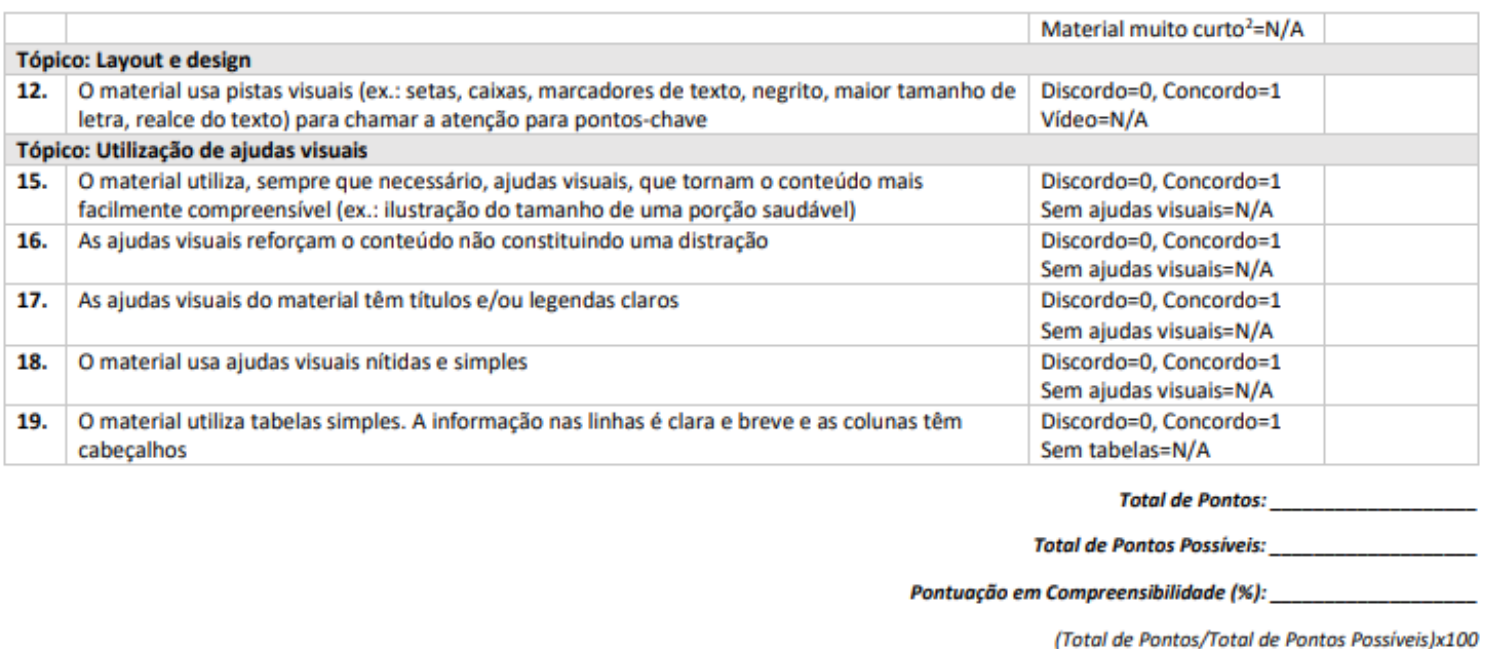

Quadro 4. - PEMAT - Materiais impressos: Acionabilidade

Instrumento para Avaliação de Materiais de Educação em Saúde - Versão para Materiais Impressos

\section{Acionabilidade}

\begin{tabular}{|c|c|c|c|}
\hline $\mathrm{N} . \mathrm{e}$ & Item & Opçōes de resposta & Classificação \\
\hline 20. & O material identifica pelo menos uma açăo ${ }^{3}$ que o utilizador pode fazer & Discordo $=0$, Concordo $=1$ & \\
\hline 21. & O material dirige-se diretamente o utilizador ao descrever açőes ${ }^{3}$ & Discordo $=0$, Concordo $=1$ & \\
\hline 22. & O material desdobra cada ação ${ }^{3}$ em passos explicitos e exequiveis & Discordo $=0$, Concordo $=1$ & \\
\hline 23. & $\begin{array}{l}\text { O material fornece ferramentas especificas (ex.: um plano de refeiç̋̄es, uma lista) sempre que } \\
\text { possam ajudar o utilizador a realizar açס̋es }{ }^{3}\end{array}$ & $\begin{array}{l}\text { Discordo }=0, \text { Concordo }=1 \\
\text { Sem ferramentas }=\mathrm{N} / \mathrm{A}\end{array}$ & \\
\hline 24. & O material apresenta instruções simples ou exemplos de como realizar cálculos & $\begin{array}{l}\text { Discordo }=0, \text { Concordo }=1 \\
\text { Sem cálculos }=\mathrm{N} / \mathrm{A}\end{array}$ & \\
\hline 25. & $\begin{array}{l}\text { Aos quadros, gráficos, tabelas ou diagramas apresentados estão associadas explicaçōes, que } \\
\text { auxiliam na realização de açōes }{ }^{3}\end{array}$ & $\begin{array}{l}\text { Discordo }=0, \text { Concordo }=1 \\
\text { Sem quadros, gráficos, } \\
\text { tabelas, diagramas }=\text { N/A }\end{array}$ & \\
\hline 26. & $\begin{array}{l}\text { O material utiliza ajudas visuais, sempre que estas tornem mais fácil, realizar uma ação }{ }^{3} \text { de } \\
\text { acordo com as instruçōes ou informação apresentadas }\end{array}$ & $\begin{array}{l}\text { Discordo }=0, \text { Concordo }=1 \\
\text { Sem ajudas visuais }=N / A\end{array}$ & \\
\hline
\end{tabular}

(Total de Pontos/Total de Pontos Possiveis) x100

${ }^{3}$ Como ação entende-se um comportamento ou instrução recomendada

Fonte: Elaboração própria.

E finalizadas as versões para materiais audiovisuais (Quadro 5) do instrumento PEMAT traduzido a adaptado para Portugal. 
Quadro 5-Materiais audiovisuais: Compreensibilidade e Acionabilidade

\section{Tópico: Conteúdo}

Tópico: Conteúdo

Tópico: Estilo e escolha de palavras

3. O material utiliza linguagem comum, do dia a dia

4. Os termos médicos utilizados săo necessários para compreender o tema apresentado no $\begin{aligned} & \text { Discordo }=0, \text { Concordo }=1 \\ & \text { 3iscordo }=0, \text { Concordo }=1\end{aligned}$ material, sendo definidos inicialmente

5. O material usa voz ativa ${ }^{1}$

Tópico: Organizaçăo

8. O material reparte a informação em pequenas secç̃̃es

9. As secç̄os do material têm cabeçalhos informativos

10. O material apresenta a informação em sequência lógica

11. O material contém um resumo

Discordo $=0$, Concordo $=1$

Discordo $=0$, Concordo $=1$ Material muito curto $^{2}=\mathrm{N} / \mathrm{A}$ Discordo $=0$, Concordo $=1$

Material muito curto $^{2}=\mathrm{N} / \mathrm{A}$

Discordo $=0$, Concordo $=1$

Discordo $=0$, Concordo $=1$

Material muito curto $^{2}=\mathrm{N} / \mathrm{A}$

Tópico: Layout e design

12. O material usa pistas visuais (ex.: setas, caixas, marcadores de texto, negrito, maior tamanho de Discordo $=0$, Concordo $=1$ letra, realce do texto) para chamar a atenção para os pontos-chave

13. O texto no ecrã é fácil de ler

14. O material permite que o utilizador oiça as palavras de forma clara (ex: com velocidade, volume e clareza sonoras adequadas à sua compreensão)

Video $=\mathrm{N} / \mathrm{A}$

Discordo $=0$, Concordo $=1$

Sem texto ou todo o texto

é narrado $=\mathrm{N} / \mathrm{A}$

Discordo $=0$, Concordo $=1$

Tópico: Utilização de ajudas visuais

Na voz ativa, o sujeito pratica a ação expressa pelo verbo. Assim, a pessoa é abordada diretamente (Ex.: Coma frutas e verduras)

${ }^{2}$ Um material muito curto é definido como um video ou apresentaçăo multimédia com menos de 1 minuto, ou um material multimédia com 6 ou menos slides/imagens

Fonte: Elaboração própria

(continuação do Quadro 5)

Instrumento para Avaliaçăo de Materiais de Educaçăo em Saúde - Versão para Materiais Audiovisuais

18. O material usa ajudas visuais nitidas e simples (ex.: com qualidade e focagem adequadas à sua compreensão)

19. O material utiliza tabelas simples. A informação nas linhas é clara e breve e as colunas têm cabeçalhos

Discordo $=0$, Concordo $=1$ Sem ajudas visuais $=N / A$ Discordo $=0$, Concordo $=1$ Sem tabelas $=\mathrm{N} / \mathrm{A}$

Total de Pontos:

Total de Pontos Possiveis:

Pontuação em Compreensibilidade (\%):

(Total de Pontos/Total de Pontos Possiveis)x100

\section{Acionabilidade}

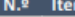

20. O material identifica pelo menos uma ação $0^{3}$ que o utilizador pode fazer

21. O material dirige-se diretamente ao utilizador ao descrever aç̋es ${ }^{3}$

22. O material decompõe cada ação $0^{3}$ em passos explicitos e exequiveis

25. Aos quadros, gráficos, tabelas ou diagramas apresentados estão associadas explicaçōes, que

auxiliam na realização de aç̃̃es ${ }^{3}$

Discordo $=0$, Concordo $=1$

Discordo $=0$, Concordo $=1$

Discordo $=0$, Concordo $=1$

Sem quadros, gráficos,

tabelas, diagramas $=N / A$

Total de Pontos:

Total de Pontos Possiveis:

(Total de Pontos/Total de Pontos Possiveis)x100 


\section{Discussão de resultados conclusão}

A existência de ferramentas que otimizem e sistematizem a prestação de cuidados de saúde são uma mais-valia para a prática profissional dos PS. Sabe-se que seguir um modelo com etapas específicas, instruções práticas e com metas atingíveis, contribui para a eficácia dos programas em cuidados de saúde (Almeida, 2019; Vaz de Almeida, 2016). Em Portugal é significativa a escassez de instrumentos formais e objetivos disponíveis para a avaliação de MES.

Ao contrário de outras realidades, em Portugal não é frequente a utilização de instrumentos que permitam avaliar a adequação de um MES ao perfil dos utentes. De acordo com Rosendo e Santiago (2017), “nos EUA é recomendado que os folhetos tenham uma legibilidade entre o $6^{\circ}$ e $9^{\circ}$ ano, 41 de acordo com a literacia nacional que, ainda assim, tem demonstrado ser superior à portuguesa (p. 248).

Nem sempre a simplificação de folhetos é suficiente para melhorar a compreensão, pelo que seria desejável desenvolver procedimentos para avaliar a inteligibilidade dos folhetos (Rosendo \& Santiago, 2017), que controlassem simultaneamente o efeito das variações da LS e avaliassem a aquisição dos conhecimentos adquiridos por meio da leitura dos mesmos. Desta forma conseguia-se perceber mais diretamente o que interessa e se os folhetos se traduzem em conhecimentos adquiridos e funcionais.
Nos Estados Unido os PS têm à sua disposição um amplo conjunto de testes formais para a avaliação de MES. Em Portugal, uma das formas que os investigadores e profissionais de saúde têm encontrado para colmatar este problema, é através da tradução de instrumentos já disponíveis noutras línguas, mas com as essenciais devidas adaptações culturais. A tradução e a adaptação de instrumentos estrangeiros poderá ser uma forma expedita e prática, porém os procedimentos adotados neste processo devem ser criteriosos e cuidadosos, uma vez que a tradução e a adaptação são tão importantes quanto a sua construção e adequação ao contexto onde vai ser aplicado. É necessário ainda que sejam realizados estudos de avaliação das propriedades psicométricas do instrumento obtido.

A limitação ao presente trabalho relaciona-se com o seu Preteste, pois de acordo com a metodologia utilizada, são necessários cerca de 30 a 40 participantes para obter a equivalência da versão adaptada. Nesta investigação tivemos um reduzido tamanho da amostra, com apenas 6 participantes, mas todos eles peritos e conhecedores destas matérias e especialistas em comunicação em saúde ou literacia em saúde

Espera-se que esta limitação seja ultrapassada em desenvolvimentos futuros, já que se pretende alargar o Preteste, bem como realizar a avaliação das propriedades psicométricas do instrumento, nomeadamente a sua validade e fiabilidade, fundamentais para obter um instrumento equivalente ao original. 
Para auxiliar a utilização do PEMAT é recomendada a leitura do Guia de Utilização, que potencia a familiaridade com os diferentes itens avaliados, aumentando a confiança e segurança na utilização do instrumento. Assim, outro dos desenvolvimentos futuros será a tradução para português do Guia de Utilização, já havendo autorização formal por parte dos autores para esse processo.

Seria ainda interessante, poder determinar o impacto na prática profissional e nos níveis de LS, após a utilização do instrumento. Esperase, que a utilização do instrumento suporte a eficácia das intervenções em LS, apontadas em estudos que utilizam o instrumento original.

\section{Conclusão}

A qualidade de comunicação dos PS é um fator que influencia a LS, e apesar da crescente consciência deste problema em Portugal, muito há a fazer neste domínio, nomeadamente junto dos PS que devem ser responsabilizados pela informação que transmitem às pessoas (Carmo, 2016).

Os PS são responsáveis pelo desenvolvimento de MES sendo importante avaliar a legibilidade, compreensibilidade e acionabilidade dos mesmos para maximizar a sua utilização, através da correspondência, com o nível de LS das pessoas a quem se destinam (Keçeci, Toprak, \& Seçil, 2017).

$\mathrm{O}$ presente trabalho abordou o processo de tradução e adaptação transcultural do instrumento PEMAT para Portugal. É um instrumento inovador no contexto português, que acrescenta valor, não só por permitir uma comunicação mais eficaz e adaptada aos níveis de LS das pessoas, mas também, pela sua utilização que de acordo com a literatura é simples e prática, estandardizada, sem custos e passível de ser replicada. A utilização da versão portuguesa do PEMAT, pode melhorar a LS das pessoas pela capacitação dos PS já que poderão selecionar e produzir MES de alta qualidade. O PEMAT poderá ajudar a desenvolver e disseminar informação em saúde com segurança, nomeadamente nas vertentes da compreensibilidade e acionabilidade. No processo de desenvolvimento da versão portuguesa do PEMAT, apresentaram-se como limitações a pequena dimensão da amostra e a pouca diversificação da mesma, esperando-se colmatar essas falhas em desenvolvimentos futuros, pois são requisitos fundamentais para obter um instrumento equivalente ao original. Outro desenvolvimento futuro será a tradução do Guia de Utilização do mesmo, que potencializa a utilização dos instrumentos pelos PS. 
Almeida, C.V., Moraes, L.K., \& Brasil, V.V. (2020). 50 Técnicas de literacia em saúde na prática. Um guia para a saúde. Alemanha: Novas Edições Académicas. Disponível em: https://www.morebooks.de/store/es/book/50-t\%C3\%A9cnicas-literacia-em-sa\%C3\%BAde-napr\%C3\%A1tica/isbn/978-620-2-55882-2

Almeida, C. V. (2019). Modelo de comunicação em saúde ACP: As competências de comunicação no cerne de uma literacia em saúde transversal, holística e prática. In C.

Lopes \& C. V. Almeida (Coords.), Literacia em saúde na prática (pp. 43-52). Lisboa: Edições ISPA [ebook] Disponível em: http://loja.ispa.pt/produto/literacia-em-saude- na- pratica

American Diabetes Associations. (2019). Standart of medical care in diabetes 2019. Diabetes care,42(1). ISSN:0149-5992.

Baker, D. W., Wolf, M. S., Feinglass, J., \& Thompson, J. A. (2008). Health literacy, cognitive ability and mortality among elderly persons. Journal of General Internal Medicine, 23(6), 723726. Doi: 10.1007/s11606-008-0566-4.

Bohanny, W., Wu, S., Liu, C., Yeh, S., Tsay, S., \& Wang, T. (2013). Health literacy, self-efficacy, and self-care behaviors in patients with type 2 diabetes mellitus. Journal of the American Association of Nurse Practitioners, OO(2013), 1-8. Doi: 10.1111/1745-7599.1207.

Campos, M., Monteiro, J., \& Ornelas, A. (2000). Fatores que afetam o consumo alimentar e a nutrição do idoso. Revista Nutrição. 3(3). Acedido em: 07-08-2019. Disponível em: http://www.scielo.br/scielo.php?script=sci_arttext\&pid=S141552732000000300002.

Canadian Council on Learning (2007). Health literacy in Canada: Initial results from the international adults literacy and skils survey. Canada: Canadian Council on Learning Acedido em 05-03-2019. Disponível em: https://www.researchgate.net/publication/317434146_Health_Literacy_in_Canada_A_Healthy_ Understanding.

Cândido, J. A. B. et al. (2017). Findrisk: diabetes mellitus risk stratification in community health. Revista Brasileira Promoção saúde, 30(3), 1-8. Acedido em: 30-07-2019. Disponível em: https://pesquisa.bvsalud.org/portal/resource/pt/biblio-876328?lang=es

Cavanaugh, K. (2012). Health Literacy in diabetes care: explanation, evidence and equipment. HHS Public Access, 1(2), 191-199. DOI: 10.2217/dmt. 11.5.

Davey, J., Holden, C. A., \& Smith, B. J. (2015). The correlates of chronic disease-related health literacy and its components among men: a systematic review. BMC Public Health, 15(589). Doi: 10.1186/s12889-015-1900-

DGS - Direção-Geral da Saúde (2008). Programa nacional e controlo da diabetes. Lisboa: Direção-geral da saúde.

DGS - Direção-Geral da Saúde (2017). Programa Nacional para a Diabetes 2017. Lisboa. Direção-geral da saúde.

DGS - Direção-Geral da Saúde (2019). Plano de Ação para a Literacia em Saúde. Lisboa. Direção-geral da saúde.

DGS - Direção-Geral da Saúde. (2004). Programa nacional para a saúde das pessoas idosas. Lisboa. Direção-geral da saúde.

DGS - Direção-Geral da Saúde. (2016). A saúde dos portugueses. Lisboa. Direção-geral da saúde. Gazmararian, J.A., Wolf, M.S., \& Baker, D. W. (2005). Health Literacy and functional health status among older adults. Archives of internal medicine,165 (17) 1946-1947. Doi: 10.1001/archinte.165.17.1946.

Huizinga, M. M., Carliste, A. J., Cavanaugh, K. L., Davis, D. L., Gregory, R. P., Schlundt, D. G., \& Rothman, R. L. (2009). Literacy, numeracy, and portion- size estimation skills. American Journal of Preventive Medicine, 36(4), 324-328. Doi: 10.1016/j.amepre.2008.11.012.

Jaana, L., et al (2001). The Finnish diabetes prevention study (DPS). Diabetes Care, 26(12), 3230-3236. Doi: 10.2337/diacare.26.12.3230.

Jenum, A.K., et al. (2006). Promoting physical activity in allow-income multiethnic district: effects os a Community intervention Study to reduce risk factors for type 2 diabetes: a Community intervention reducing inactivity. Diabetes Care, 29(7), 1605-1612.DOI:10.2337/dc05-1587.

Kim, S. (2009). Health literacy and functional health status in Korean old adults. Journal of Clinical Nursing, 18, 2337-2343. Doi:10.1111/j.1365-2702.2008.02739. x. 
Almeida, C.V., Moraes, L.K., \& Brasil, V.V. (2020). 50 Técnicas de literacia em saúde na prática. Um guia para a saúde. Alemanha: Novas Edições Académicas. Disponível em: https://www.morebooks.de/store/es/book/50-t\%C3\%A9cnicas-literacia-em-sa\%C3\%BAde-napr\%C3\%A1tica/isbn/978-620-2-55882-2

Almeida, C. V. (2019). Modelo de comunicação em saúde ACP: As competências de comunicação no cerne de uma literacia em saúde transversal, holística e prática. In C.

Lopes \& C. V. Almeida (Coords.), Literacia em saúde na prática (pp. 43-52). Lisboa: Edições ISPA [ebook] Disponível em: http://loja.ispa.pt/produto/literacia-em-saude- na- pratica

American Diabetes Associations. (2019). Standart of medical care in diabetes 2019. Diabetes care,42(1). ISSN:0149-5992.

Baker, D. W., Wolf, M. S., Feinglass, J., \& Thompson, J. A. (2008). Health literacy, cognitive ability and mortality among elderly persons. Journal of General Internal Medicine, 23(6), 723726. Doi: 10.1007/s11606-008-0566-4.

Bohanny, W., Wu, S., Liu, C., Yeh, S., Tsay, S., \& Wang, T. (2013). Health literacy, self-efficacy, and self-care behaviors in patients with type 2 diabetes mellitus. Journal of the American Association of Nurse Practitioners, OO(2013), 1-8. Doi: 10.1111/1745-7599.1207.

Campos, M., Monteiro, J., \& Ornelas, A. (2000). Fatores que afetam o consumo alimentar e a nutrição do idoso. Revista Nutrição. 3(3). Acedido em: 07-08-2019. Disponível em: http://www.scielo.br/scielo.php?script=sci_arttext\&pid=S141552732000000300002.

Canadian Council on Learning (2007). Health literacy in Canada: Initial results from the international adults literacy and skils survey. Canada: Canadian Council on Learning Acedido em 05-03-2019. Disponível em: https://www.researchgate.net/publication/317434146_Health_Literacy_in_Canada_A_Healthy_ Understanding.

Cândido, J. A. B. et al. (2017). Findrisk: diabetes mellitus risk stratification in community health. Revista Brasileira Promoção saúde, 30(3), 1-8. Acedido em: 30-07-2019. Disponível em: https://pesquisa.bvsalud.org/portal/resource/pt/biblio-876328?lang=es

Cavanaugh, K. (2012). Health Literacy in diabetes care: explanation, evidence and equipment. HHS Public Access, 1(2), 191-199. DOI: 10.2217/dmt. 11.5.

Davey, J., Holden, C. A., \& Smith, B. J. (2015). The correlates of chronic disease-related health literacy and its components among men: a systematic review. BMC Public Health, 15(589). Doi: 10.1186/s12889-015-1900-

DGS - Direção-Geral da Saúde (2008). Programa nacional e controlo da diabetes. Lisboa: Direção-geral da saúde.

DGS - Direção-Geral da Saúde (2017). Programa Nacional para a Diabetes 2017. Lisboa. Direção-geral da saúde.

DGS - Direção-Geral da Saúde (2019). Plano de Ação para a Literacia em Saúde. Lisboa. Direção-geral da saúde.

DGS - Direção-Geral da Saúde. (2004). Programa nacional para a saúde das pessoas idosas. Lisboa. Direção-geral da saúde.

DGS - Direção-Geral da Saúde. (2016). A saúde dos portugueses. Lisboa. Direção-geral da saúde. Gazmararian, J.A., Wolf, M.S., \& Baker, D. W. (2005). Health Literacy and functional health status among older adults. Archives of internal medicine,165 (17) 1946-1947. Doi: 10.1001/archinte.165.17.1946.

Huizinga, M. M., Carliste, A. J., Cavanaugh, K. L., Davis, D. L., Gregory, R. P., Schlundt, D. G., \& Rothman, R. L. (2009). Literacy, numeracy, and portion- size estimation skills. American Journal of Preventive Medicine, 36(4), 324-328. Doi: 10.1016/j.amepre.2008.11.012.

Jaana, L., et al (2001). The Finnish diabetes prevention study (DPS). Diabetes Care, 26(12), 3230-3236. Doi: 10.2337/diacare.26.12.3230.

Jenum, A.K., et al. (2006). Promoting physical activity in allow-income multiethnic district: effects os a Community intervention Study to reduce risk factors for type 2 diabetes: a Community intervention reducing inactivity. Diabetes Care, 29(7), 1605-1612.DOI:10.2337/dc05-1587.

Kim, S. (2009). Health literacy and functional health status in Korean old adults. Journal of Clinical Nursing, 18, 2337-2343. Doi:10.1111/j.1365-2702.2008.02739. x. 